\title{
Production and Characterization of a Monoclonal Antibody Raised Against Surface Antigens from Mycelium of Gaeumannomyces graminis var. tritici: Evidence for an Extracellular Polyphenol Oxidase
}

\author{
Christopher R. Thornton, Frances M. Dewey, and Christopher A. Gilligan
}

First and third authors: Department of Plant Sciences, University of Cambridge, Downing Street, Cambridge, UK, CB2 3EA; second author: Department of Plant Sciences, University of Oxford, South Parks Road, Oxford, UK, OX1 3RB.

Accepted for publication 14 October 1996.

\begin{abstract}
Thornton, C. R., Dewey, F. M., and Gilligan, C. A. 1997. Production and characterization of a monoclonal antibody raised against surface antigens from mycelium of Gaeumannomyces graminis var. tritici: Evidence for an extracellular polyphenol oxidase. Phytopathology 87:123-131.

A murine monoclonal antibody (MAb) of immunoglobulin class $\mathrm{M}$ (IgM) was raised against surface antigens from Gaeumannomyces graminis var. tritici and, by enzyme-linked immunosorbent assay, recognized isolates of $G$. graminis var. tritici, G. graminis var. avenae and $G$. graminis var. graminis. Characterization of the antigen by heat and protease treatments showed that the epitope recognized by the MAb was a protein. Antigen production was detected only in live mycelia. Immunofluorescence studies showed that the antigen was associated with both the broad melanized macrohyphae and hyaline mycelia of G. graminis var. tritici. Secretion of antigen into an aqueous minimal medium was promoted only by exposure of live mycelia to certain phenolic substrates, including monophenols ortho-, para-, and meta-cresol; 3,4,5-trihydroxybenzoic acid (gallic acid); and phenolic amino acid L-3-(3,4-dihydroxy-

phenyl) alanine (L-DOPA). Antigen secretion was not promoted by 3-(4hydroxyphenyl) alanine (L-tyrosine). The MAb reacted strongly with purified enzyme laccase (polyphenol oxidase, EC 1.10.3.2) but did not recognize purified tyrosinase (monophenol oxidase, EC 1.14.18.1). Moreover, chemicals that bind to copper and inhibit copper-containing enzymes such as laccase completely inhibited antigen secretion in response to L-DOPA. The MAb was tested for specificity against a wide range of fungi, common yeast species, and gram positive and negative bacteria. It did not recognize antigens from a broad range of unrelated fungi, including Gliocladium roseum, Fusarium sp., Phoma exigua, Phialophora fastigiata, Penicillium crustosum, Pythium ultimum, Rhizopus stolonifer, Rhizoctonia carotae, $R$. oryzae, $R$. tuliparum, and Trichoderma viride, nor did it recognize surface antigens from yeasts or bacteria. The MAb cross-reacted with antigens from Botrytis spp., Chaetomium globosum, $R$. cerealis, and $R$. solani. However, secretion of antigen by $R$. solani and $R$. cerealis was not promoted by L-DOPA, and secretion by $C$. globosum in response to the phenolic amino acid was significantly less compared to G. graminis var. tritici.
\end{abstract}

The fungus Gaeumannomyces graminis (Sacc.) Von Arx \& D. Olivier var. tritici J. Walker causes take-all, a major root disease of cultivated wheat that is distributed throughout the world in both northern and southern temperate zones. The soilborne fungal pathogen can exist saprophytically on plant debris in soil or parasitically on susceptible cereal crop or weed hosts (37). G. graminis var. tritici has been extensively investigated for many years, but control remains elusive. Because of this, take-all remains one of the most important factors limiting cereal production in many countries.

The search for effective disease control has been hampered, in part, by the absence of quick, sensitive, and specific assays for detection and quantification of G. graminis var. tritici. Conventional methods for detecting G. graminis var. tritici, involving pathogenicity tests and production of perithecia on host tissue, are notoriously slow and laborious. Furthermore, because G. graminis var. tritici typically does not sporulate in culture, correct identification relies on cultural characteristics, such as pigmentation, thick runner hyphae, and hyphal curling at the colony margins (2). Wheat roots infected with $G$. graminis var. tritici also commonly harbor fast-growing Pythium, Fusarium, and Rhizoctonia spp., which can interfere with recovery of the take-all pathogen. Several selective media have been developed to aid in recovery and diagnosis of G. graminis var. tritici from infected plant material $(13,21)$,

Corresponding author: C. R. Thornton; E-mail address: crt20@cus.cam.ac.uk

Publication no. P-1996-1209-01R

(C) 1997 The American Phytopathological Society and although these have undoubtedly improved the recovery rate of the target organism, their routine use is restrained by their laborintensive nature.

Molecular techniques have considerable potential for detection and quantification of soilborne fungi. Nucleic acid-based techniques, such as DNA probes, polymerase chain reaction (PCR), and restriction length polymorphisms, have been used to detect and differentiate $G$. graminis var. tritici and related fungi from other fungal species in planta and to detect the pathogen in soil $(18,19$, 27 ), but these methods are unable to differentiate between live and dead fungal biomasses. Hybridoma technology allows the development of monoclonal antibodies (MAbs) that can be used to develop quick, sensitive, and specific assays that detect only live, actively growing fungal biomasses $(32,33)$.

In this paper, we describe the production and characterization of a MAb raised against surface antigens of $G$. graminis var. tritici. We show that secretion of the antigen by live mycelia of G. graminis var. tritici is promoted only by certain phenolic compounds, including the phenolic amino acid L-3-(3,4-dihydroxyphenyl) alanine (L-DOPA). We present evidence showing that the antigen recognized by the MAb is the extracellular enzyme laccase and discuss how the MAb may be used to differentiate between $G$. graminis and other fungi.

\section{MATERIALS AND METHODS}

Fungal culture. MAbs were raised against an isolate of $G$. graminis var. tritici (referred to as GP20) obtained from G. L. Bateman, Institute of Arable Crop Research, Harpenden, England. 
Organisms used for screening antibodies (Table 1) were maintained on half-strength potato dextrose agar (PDA) (fungi and yeasts) or Luria agar (bacteria) either in petri dishes or on slants. G. graminis isolates were grown at $20^{\circ} \mathrm{C}$, and Rhizoctonia isolates were grown at $17^{\circ} \mathrm{C}$. All remaining organisms were grown at $23^{\circ} \mathrm{C}$.

Development of MAbs, preparation of immunogen, and immunization regime. One-week-old PDA slant cultures of GP20 were washed with $2 \mathrm{ml}$ of phosphate-buffered saline (PBS: $0.8 \%$ $\mathrm{NaCl} ; 0.02 \% \mathrm{KCl} ; 0.115 \% \mathrm{Na}_{2} \mathrm{HPO}_{4} ; 0.02 \% \mathrm{KH}_{2} \mathrm{PO}_{4} ; \mathrm{pH} 7.2$ ). The surface washings were centrifuged in $1.5-\mathrm{ml}$ microcentrifuge tubes at $12,000 \times g$ for $10 \mathrm{~min}$ to remove cell debris, and the supernatant was transferred to a clean tube for use as immunogen.

Three 6-week-old BALB/c female white mice were each given three intraperitoneal injections (300 $\mu \mathrm{l}$ per injection) of fresh immunogen at 2-week intervals. The mice each received Quil A adjuvant (Superfos a/s, Vedbaek, Denmark) at a concentration of 33 $\mu \mathrm{g}$ per dose per injection.
Production and screening of hybridomas and determination of antibody specificities. Hybridoma cells were produced by the method described by Dewey et al. (10), and the supernatants were screened by enzyme-linked immunosorbent assay (ELISA). Microtiter wells (Labsystems 952107, Helsinki, Finland) arranged in strips were coated overnight at $4^{\circ} \mathrm{C}$ with cell-free PBS surface washings from 1-week-old PDA slant cultures of fungal isolates diluted 10-fold in PBS. After an overnight antigen immobilization step at $4^{\circ} \mathrm{C}$ the wells were washed four times with PBST (PBS and $0.05 \%$ [vol/vol] Tween 20) and once each with PBS and reverse osmosis water (R.O. $\left.\mathrm{H}_{2} \mathrm{O}\right)$ and air-dried at room temperature (RT; $23 \pm 2{ }^{\circ} \mathrm{C}$ ) in a laminar flow hood. The strips were stored in sealed plastic bags at $4^{\circ} \mathrm{C}$ in preparation for screening of hybridoma supernatants by ELISA as described below.

ELISA formats. Preliminary screening. Preliminary screening of hybridoma supernatants was performed by ELISA, using microtiter wells coated with 10-fold dilutions of PBS surface washings

TABLE 1. Details of fungal cultures

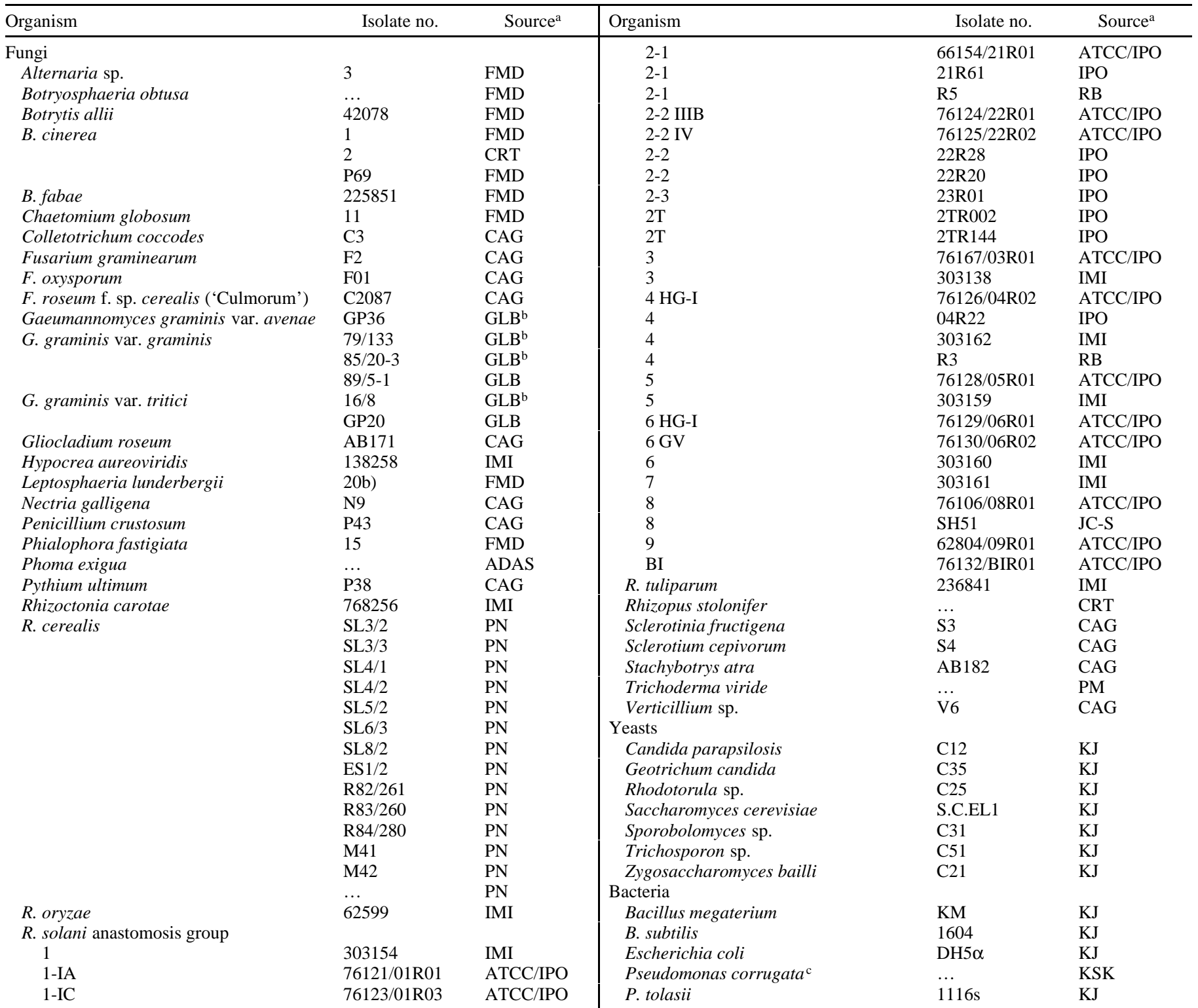

${ }^{a}$ CRT: C. R. Thornton; FMD: F. M. Dewey; CAG: C. A. Gilligan; GLB: G. L. Bateman, AFRC Inst. Arable Crops Res. Exp. Stn., Harpenden, England; PN: P. Nicholson, Cambridge Lab., John Innes Centre Plant Sci. Res., Norwich, England; RB: R. Baker, Dep. Plant Pathol. Weed Sci., Colo. State Univ.; PM: P. Mills, Hortic. Res. Int., Wellesbourne, England; KJ: K. Johnstone, Dep. Plant Sci., Univ. Cambridge; KSK: K. S. Killham, Dep. Plant Soil Sci., Univ. Aberdeen, Scotland; IMI: Int. Mycol. Inst., Egham, England; ADAS: Agric. Devel. Advisory Serv., Cambridge; IPO: Inst. voor Planteziektenk. Onderzoek, Wageningen, the Netherlands.

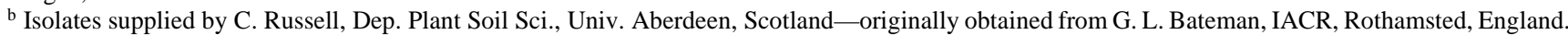

${ }^{c}$ This isolate was transformed with the lux operon from Vibrio fischera. 
from 1-week-old PDA slant cultures of GP20. The assay procedure was as follows. Wells containing immobilized antigen were incubated successively with hybridoma supernatant for $1 \mathrm{~h}$ and then by commercial goat anti-mouse polyvalent (immunoglobulin classes IgG, IgA, and IgM) peroxidase conjugate (A0412; Sigma Chemical Co., Poole, Dorset, England) diluted 1:1,000 in PBST for another hour. Bound antibody was visualized by incubating wells with tetramethyl benzidine (TMB) substrate solution for 30 min, reactions were stopped by the addition of $3 \mathrm{M} \mathrm{H}_{2} \mathrm{SO}_{4}$, and absorbance values were determined with an EL311sx automated microplate reader (Bio-Tek Instruments, Inc., Winooski, VT). Wells were given four 5-min rinses with PBST between incubations. Working volumes were $50 \mu \mathrm{l}$ per well, and control wells were incubated with tissue culture medium (TCM) containing $10 \%$ (vol/vol) fetal calf serum. All incubation steps were performed at RT in sealed plastic bags.

Secondary screening. Hybridoma cell lines selected on the basis of recognition of GP20 antigens were tested further by ELISA for specificity against surface washings diluted 1:10 from 1-week-old PDA slant cultures of GP20 and other related and unrelated fungi and with soluble antigens from 2- and 1-day-old slant cultures of yeasts and bacteria respectively (Table 1). Soluble antigens from yeasts and bacteria were prepared according to the method for fungal surface washings. The ELISA format (format I) employed in this secondary screening procedure was as follows. Wells containing immobilized antigen were blocked for 30 min with $100 \mu \mathrm{l}$ of Tris-buffered saline containing $0.3 \%(\mathrm{wt} / \mathrm{vol})$ casein $(\mathrm{TBS} / \mathrm{c}: 8 \mathrm{~g}$ of $\mathrm{NaCl}, 3 \mathrm{~g}$ of Tris, and $0.2 \mathrm{~g}$ of $\mathrm{KCl}$ per liter of R.O. $\mathrm{H}_{2} \mathrm{O}$, adjusted to $\mathrm{pH} 8.3$ with $1 \mathrm{~N} \mathrm{HCl}$ ). Wells were incubated successively with MAb supernatant for $1 \mathrm{~h}$, followed by commercial goat anti-mouse polyvalent (IgG, IgA, and IgM) peroxidase conjugate (A0412) diluted 1:1,000 in TBS/c for another hour. Bound antibody was visualized by incubating wells with TMB substrate solution for $30 \mathrm{~min}$, reactions were stopped by the addition of $3 \mathrm{M}$ $\mathrm{H}_{2} \mathrm{SO}_{4}$, and absorbance values were determined with an EL311sx automated microplate reader. Wells were given four 5-min rinses with TBS/c between incubations and a final rinse with R.O. $\mathrm{H}_{2} \mathrm{O}$ prior to the addition of substrate. Working volumes were $50 \mu \mathrm{l}$ per well, and control wells were incubated with TCM containing $10 \%$ ( $\mathrm{vol} / \mathrm{vol}$ ) fetal calf serum. All incubation steps were performed at RT in sealed plastic bags. An additional ELISA format (format II) was employed as described above, but the incubation periods of primary antibody and secondary antibody-peroxidase conjugate were each reduced to $15 \mathrm{~min}$, and substrate time was reduced to 5 $\min$.

Determination of Ig subclass and cloning procedure. The Ig subclass of MAbs from selected cell lines was determined with a commercial mouse MAb isotyping kit according to the manufacturers instructions (ISO-1; Sigma). Hybridoma cell lines were cloned by limiting dilution, and selected cell lines were grown in bulk in a nonselective medium, preserved by slowly freezing in fetal bovine serum/dimethyl sulfoxide ( $92: 8$ [vol/vol]), and maintained in liquid nitrogen.

Antigen characterization and pronase and trypsin digestion of immobilized antigen. After washing, antigens (GP20 surface washings diluted 1:10) immobilized in microtiter wells (50 $\mu \mathrm{l})$ were incubated with pronase (Protease XIV) (P5147; Sigma) (0.25 units per well) or trypsin (T4424; Sigma) solution (1 mg ml${ }^{-1}$ in PBS) at $37^{\circ} \mathrm{C}$ for the appropriate time period and washed three times with PBS. Wells incubated with trypsin were treated for $10 \mathrm{~min}$ with a $0.1 \mathrm{mg} \mathrm{ml}^{-1}$ solution of trypsin inhibitor (T6522; Sigma) and given three more washes with PBS. Washed wells were blocked. Controls received PBS without pronase or trypsin and inhibitor but were otherwise treated similarly. The wells were assayed by ELISA format I with half-strength DD8 MAb.

Boiling. Cell-free surface washings from a 1-week-old PDA slant culture of GP20 washed with $2 \mathrm{ml}$ of PBS were boiled for the appropriate period (up to $1 \mathrm{~h}$ ), cooled, and centrifuged at
$12,000 \times \mathrm{g}$ for $5 \mathrm{~min}$. Samples were diluted with PBS to give $1 / 10$-strength surface washings and transferred to microtiter wells. Control samples were incubated at RT but were otherwise treated similarly. After immobilization, wells were washed and assayed by ELISA format I with half-strength DD8 MAb.

Periodate oxidation of immobilized antigen. Immobilized antigens were treated with $50 \mu \mathrm{l}$ of sodium metaperiodate $(20 \mathrm{mM}$ $\mathrm{NaIO}_{4}$ in $50 \mathrm{mM}$ sodium acetate buffer, pH 4.5) (S1878; Sigma), whereas control wells received only buffer. After incubation for the appropriate period in darkness at $4^{\circ} \mathrm{C}$, wells were washed three times with PBS and assayed by ELISA format I with half-strength DD8 MAb.

Promotion of antigen secretion by phenolic substrates. Selected phenolic substrates (Table 2) were added separately to unsterilized minimal medium $\left(5.0 \mathrm{~g}\right.$ of $\mathrm{NH}_{4} \mathrm{NO}_{3}, 14.0 \mathrm{~g}$ of $\mathrm{KH}_{2} \mathrm{PO}_{4}$, $0.5 \mathrm{~g}$ of $\mathrm{MgSO}_{4} \cdot 7 \mathrm{H}_{2} \mathrm{O}, 1.5 \mathrm{mg}$ of $\mathrm{FeSO}_{4} \cdot 7 \mathrm{H}_{2} \mathrm{O}, 0.9 \mathrm{mg}$ of $\mathrm{ZnSO}_{4}$, $0.4 \mathrm{mg}$ of $\mathrm{MnSO}_{4}, 0.4 \mathrm{mg}$ of $\mathrm{CuSO}_{4}, 10.0 \mathrm{~g}$ of D-glucose, and 0.1 $\mathrm{g}$ of rifampicin [R3501; Sigma] per liter of R.O. $\mathrm{H}_{2} \mathrm{O}$ ) at $500 \mathrm{mg}$ liter $^{-1}$ and mixed until fully dissolved. Aliquots $(10 \mathrm{ml})$ of each solution were transferred to respective $25-\mathrm{ml}$ polyvials and inoculated with single 7-cm-diameter plugs of mycelium taken from the leading edge of a 5-day-old PDA culture of G. graminis var. tritici grown at $20^{\circ} \mathrm{C}$. Inoculated solutions were incubated without shaking at $23^{\circ} \mathrm{C}$. At appropriate time intervals, 50- $\mu$ l aliquots were removed from each tube, transferred to individual microtiter wells, and after immobilization (overnight at $4^{\circ} \mathrm{C}$ ) and washing were assayed by ELISA format II with half-strength DD8 MAb. There were five replicate tubes for each phenolic compound studied and an equivalent number of replicate negative control samples that contained inoculated minimal medium only. The $\mathrm{pH}$ of solutions prior to study and after $8 \mathrm{~h}$ of incubation at $23^{\circ} \mathrm{C}$ are given in Table 2 .

Inhibition of antigen production. The effects of inhibitors on antigen production were determined by the assay system described above. Selected inhibitors (Table 2) were added at a concentration

TABLE 2. List of substrates, inhibitors, and solution pHs used in the characterization of the Gaeumannomyces graminis var. tritici (isolate GP20) DD8 antigen

\begin{tabular}{|c|c|c|}
\hline & \multicolumn{2}{|c|}{ Stock solution $\mathrm{pH}$} \\
\hline & $\begin{array}{c}\text { Before } \\
\text { incubation }\end{array}$ & $\begin{array}{l}\text { After } 8 \mathrm{~h} \text { of } \\
\text { incubation }^{\mathrm{a}}\end{array}$ \\
\hline \multicolumn{3}{|l|}{ Substrate } \\
\hline L-(+)-Ascorbic acid & 3.80 & $3.66 \pm 0.008$ \\
\hline meta-Cresol & 4.20 & $4.27 \pm 0.005$ \\
\hline ortho-Cresol & 4.23 & $4.28 \pm 0.007$ \\
\hline para-Cresol & 4.21 & $4.29 \pm 0.015$ \\
\hline $\begin{array}{l}\text { L-3-(3,4-Dihydroxyphenyl) alanine (L- } \\
\text { DOPA) (MM-LD) }\end{array}$ & 4.17 & $4.27 \pm 0.011$ \\
\hline $\begin{array}{l}\text { 3,4,5-Trihydroxybenzoic acid (gallic } \\
\text { acid) }\end{array}$ & 3.76 & $3.83 \pm 0.009$ \\
\hline $\begin{array}{l}N, N^{\prime} \text {-Bis (3,5-dimethoxy-4-hydroxyben - } \\
\text { zylidene) hydrazine (syringaldazine) }\end{array}$ & 4.22 & $4.29 \pm 0.004$ \\
\hline Tannic acid & 4.06 & $4.13 \pm 0.008$ \\
\hline 3-(4-Hydroxyphenyl) alanine (L-tyrosine) & 4.18 & $4.26 \pm 0.031$ \\
\hline Negative control $^{\mathrm{c}}$ & 4.11 & $4.28 \pm 0.005$ \\
\hline \multicolumn{3}{|l|}{ Inhibitor } \\
\hline Diethyldithiocarbamate & 4.31 & $4.41 \pm 0.007$ \\
\hline Ethylenediaminetetraacetic acid & 4.06 & $4.08 \pm 0.006$ \\
\hline Fusaric acid & 4.26 & $4.25 \pm 0.005$ \\
\hline $\begin{array}{l}\text { 2-Hydroxy-2,4,6-cycloheptatriene-1-one } \\
\text { (tropolone) }\end{array}$ & 4.18 & $4.24 \pm 0.006$ \\
\hline $\begin{array}{l}\text { 5-Hydroxy-2-(hydroxymethyl)- } 4 \mathrm{H}- \\
\text { pyran-4-one (kojic acid) }\end{array}$ & 4.21 & $4.24 \pm 0.008$ \\
\hline 2-Mercaptobenzothiazole & 4.18 & $4.24 \pm 0.004$ \\
\hline Pyroquilon & 4.19 & $4.25 \pm 0.003$ \\
\hline Negative control ${ }^{b}$ & 4.17 & $4.27 \pm 0.011$ \\
\hline Positive control $^{\mathrm{c}}$ & 4.14 & $4.28 \pm 0.005$ \\
\hline
\end{tabular}



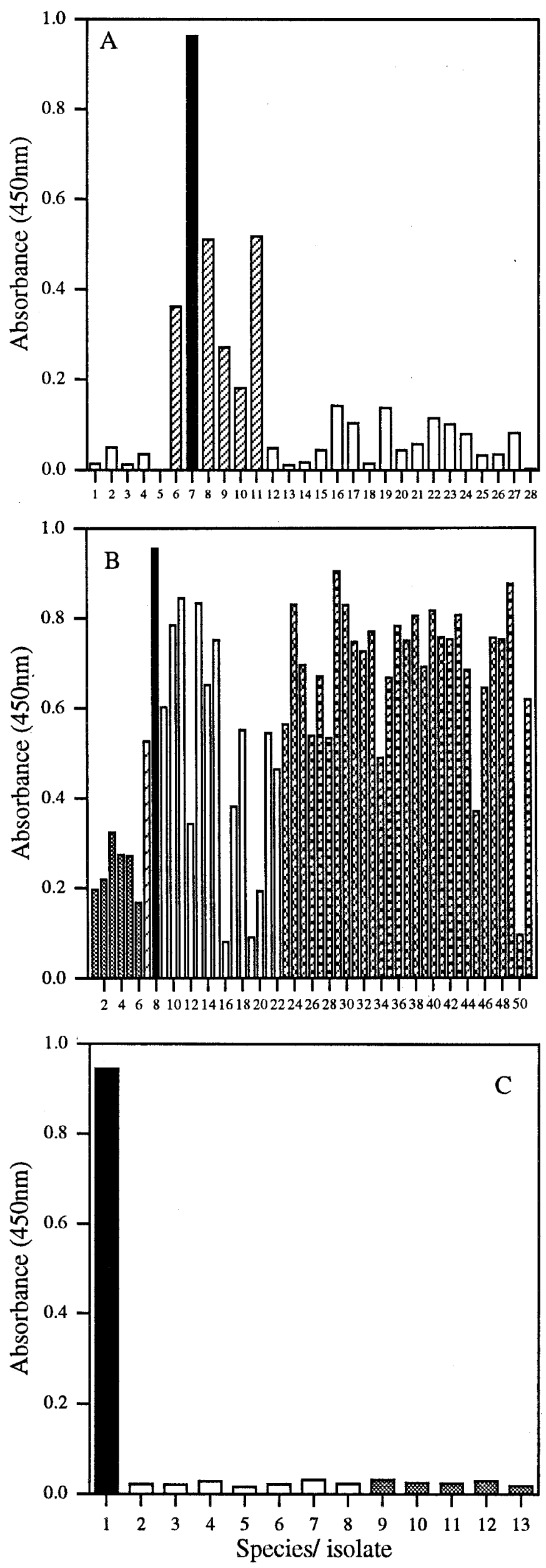

of $100 \mu \mathrm{g} \mathrm{ml}^{-1}$ to unsterilized minimal medium that contained 500 $\mathrm{mg}$ of the phenolic amino acid L-DOPA per liter (MM-LD). Four replicate $10-\mathrm{ml}$ samples of each mixture, contained in $25-\mathrm{ml}$ polyvials, were inoculated, sampled, and tested by ELISA format II as described above. The negative control was as described previously. The positive control consisted of MM-LD only. The $\mathrm{pH}$ of solutions prior to study and after $8 \mathrm{~h}$ of incubation at $23^{\circ} \mathrm{C}$ are given in Table 2.

Secretion of antigen by other organisms. Three replicate 10$\mathrm{ml}$ samples of MM-LD, contained in 25-ml polyvials, were inoculated separately with single 7-cm-diameter plugs of mycelium taken from the leading edge of 5-day-old PDA cultures of either $G$. graminis var. tritici (isolate GP20), Botrytis cinerea (isolate 2), Chaetomium globosum (isolate 11), Rhizoctonia solani (isolate 76124/22R01), or $R$. cerealis (isolate SL4/1). Each was sampled and tested by ELISA format II as described above.

Recognition of laccase. Microtiter wells were coated for $2 \mathrm{~h}$ at RT with $50 \mu \mathrm{l}$ of poly-L-lysine (10 $\mu \mathrm{g}$ of PBS per ml) (P1274; Sigma). Wells were washed three times with PBS and incubated with commercial laccase (polyphenol oxidase, EC 1.10.3.2) (L5510; Sigma) solution double-diluted in PBS for another $2 \mathrm{~h}$ at RT. The initial concentration of the stock solution was $1 \mathrm{mg}$ of solid per $\mathrm{ml}$ of PBS (1.5 $\mu \mathrm{g}$ of protein per $\mathrm{ml})$ and was prepared by thorough mixing for $4 \mathrm{~h}$ at RT, followed by centrifugation at $12,000 \times g$ for $10 \mathrm{~min}$. Control wells were prepared in the same way with a PBS solution of commercial tyrosinase (monophenol oxidase, EC 1.14.18.1) (T7755; Sigma) with an equivalent initial concentration of $1 \mathrm{mg} \mathrm{ml}^{-1}$ (105 $\mu \mathrm{g}$ of protein per $\mathrm{ml}$ ). Wells containing immobilized protein were washed three more times with PBS and were blocked for $16 \mathrm{~h}$ at $4^{\circ} \mathrm{C}$ with TBS/c (pH 8.3). Blocking solution was removed from the wells, and the wells were assayed by ELISA format I with half-strength DD8 MAb. Protein concentrations were determined by the Bio-Rad (Richmond, CA) Bradford protein assay with bovine serum albumin (BSA) as the standard.

Fig. 1. Tissue culture supernatant from hybridoma cell line DD8 tested by enzyme-linked immunosorbent assay format II against surface washings from A, Gaeumannomyces spp. and unrelated, non-cross-reactive, fungal species (isolates are given in parentheses). 1, Alternaria sp. (3); 2, Colletotrichum coccodes (C3); 3, Fusarium culmorum (C2087); 4, F. graminearum $(\mathrm{F} 2) ; 5$, F. oxysporum (F01); 6, G. graminis var. tritici (16/8); 7, G. graminis var. tritici (GP20); 8, G. graminis var. graminis (79/133); 9, G. graminis var. graminis (85/20-3); 10, G. graminis var. graminis (89/5-1); 11, G. graminis var. avenae (GP36); 12, Gliocladium roseum (AB171); 13, Hypocrea aureoviridis (138258); 14, Leptosphaeria lagerbergii (20b); 15, Nectria galligena (N9); 16, Penicillium crustosum (P43); 17, Phialophora fastigiata (15); 18, Phoma exigua; 19, Pythium ultimum (P38); 20, Rhizoctonia carotae (768256); 21, R. oryzae (62599); 22, R. tuliparum (236841); 23, Rhizopus stolonifer; 24 , Sclerotinia fructigena (S3); 25, Sclerotium cepivorum (S4); 26, Stachybotrys atra (AB182); 27, Trichoderma viride; 28, Verticillium sp. (V6). B, Gaeumannomyces graminis var. tritici (GP20) and unrelated, cross-reactive fungal species. 1, Botryosphaeria obtusa; 2, Botrytis allii (42078); 3, B. cinerea (1); 4, B. cinerea (2); 5, B. cinerea (P69); 6, B. fabae (225851); 7, Chaetomium globosum (11); 8, G. graminis var. tritici (GP20); 9 to 22, Rhizoctonia cerealis: 9, (SL3/2); 10, (SL3/3); 11, (SL4/1); 12, (SL4/2); 13, (SL5/2); 14, (SL6/3); 15, (SL8/2); 16, (ES1/2); 17, (R82/261); 18, (R83/260); 19, (R84/ 280); 20, (M41); 21, (M42); 22, (...); 23 to 51, R. solani: 23, (303154); 24, (76121/01R01); 25, (76123/01R03); 26, (66154/21R01); 27, (21R61); 28, (R5); 29, (76124/22R01); 30, (76125/22R02); 31, (22R28); 32, (22R20); 33, (23R01); 34, (2TR002); 35, (2TR144); 36, (76167/03R01); 37, (303138); 38, (76126/ 04R02); 39, (04R22); 40, (303162); 41, (R3); 42, (76128/05R01); 43, (303159); 44, (76129/06R01); 45, (76130/06R02); 46, (303160); 47, (303161); 48, (76106/ 08R01); 49, (SH51); 50, (62804/09R01); 51, (76132/BIR01). C, Gaeumannomyces graminis var. tritici (GP20) and yeasts and bacteria. 1, G. graminis var. tritici (GP20); 2, Candida parapsilopsis (C12); 3, Geotrichum candida (C35); 4, Rhodotorula (C25); 5, Saccharomyces cerevisiae (S.C.EL1); 6, Sporobolomyces (C31); 7, Trichosporon (C51); 8, Zygosaccharomyces bailli (C21); 9, Bacillus megaterium (KM); 10, B. subtilis (1604); 11, Escherichia coli (DH5 $\alpha$ ); 12, Pseudomonas corrugata; 13, P. tolasii (1116s). Each bar (isolate/species against absorbance at $450 \mathrm{~nm}$ ) represents the mean of duplicate samples. 
Indirect immunofluorescence (IF). Cultures of GP20 were grown on multiwell slides (ICN 60-408-05; ICN Biochemical, Cleveland) and embedded in PDA (7) for 7 days at $20^{\circ} \mathrm{C}$. The slides were air-dried and fixed according to Dewey (9) and blocked for 30 min with PBS containing $1.0 \%$ (wt/vol) BSA. The wells were incubated with half-strength DD8 MAb supernatant for $1 \mathrm{~h}$, followed by goat anti-mouse IgM ( $\mu$-chain specific) fluorescein isothiocyanate conjugate (F9259; Sigma) diluted 1:40 in PBST containing $0.5 \%$ (wt/vol) BSA for another hour. All incubation steps were performed at RT in a moist chamber. Control wells were incubated with half-strength TCM. Slides were given three 5-min rinses with PBST between incubations and mounted in Citifluor (University of Kent, Canterbury, England) prior to examination with a Leica (Leica Cambridge Ltd., Cambridge) TCS 4D inverse confocal microscope with a $\times 20 \mathrm{Ph} 2$ dry 0.4 numerical aperture objective. The lens was corrected for a nominal cover-glass thickness of $170 \mu \mathrm{m}$ (No. 1). Line averaging was used to reduce electronic and mechanical noise.

\section{RESULTS}

Specificity of hybridoma cell lines. A single fusion was performed. From this, 320 hybridoma cell lines were screened for $\mathrm{MAb}$ production, and all tested positive for recognition of GP20 antigens using the preliminary screening procedure. Ten of these were selected for further testing on the basis of absorbance values $\left(A_{450}>0.7\right)$. In secondary specificity screening tests, 8 of the 10 cell lines selected produced MAbs that were nonspecific, reacting strongly in ELISA against surface washings from all of the fungal isolates tested (data not shown). Of the remaining two cell lines, one stopped producing antibodies, and the other, DD8, produced a $\mathrm{MAb}$ that exhibited specificity for antigens from G. graminis isolates. This cell line was subcloned three times.

In ELISA format II, the DD8 MAb strongly recognized surface antigens from G. graminis var. tritici (GP20) (Fig. 1A). It also recognized surface antigens from $G$. graminis var. tritici isolate 16/8 and G. graminis var. graminis and avenae isolates 79/133, 85/20-3, 89/5-1, and GP36. The MAb cross-reacted with surface antigens from three Botrytis species (5 isolates) and with antigens from a single isolate of $C$. globosum and multiple isolates of $R$. cerealis (14 isolates) and $R$. solani (28 isolates) (Fig. 1B). They did not recognize antigens from a broad range of other unrelated fungi, including Gliocladium roseum, Fusarium sp., Phoma exigua, Phialophora fastigiata, Penicillium crustosum, Pythium ultimum, Rhizopus stolonifer, Rhizoctonia carotae, $R$. oryzae, $R$. tuliparum, and Trichoderma viride (Fig. 1B) nor did they recognize surface antigens from a number of common yeast species or gram positive and negative bacteria (Fig. 1C).

Isotyping. The MAb from the subcloned cell line DD8 was from Ig class $\mathrm{M}$.

Characterization of antigen and effect of heat, protease, and periodate. Surface antigens of $G$. graminis var. tritici (GP20) recognized by the DD8 MAb were heat labile. There was a sig-

TABLE 3. Absorbance values from enzyme-linked immunosorbent assay tests with periodate-treated antigens from Gaeumannomyces graminis var. tritici (isolate GP20), using DD8 immunoglobulin class M monoclonal antibodies ${ }^{\mathrm{a}}$

\begin{tabular}{lcc}
\hline & \multicolumn{2}{c}{ Absorbance $(450 \mathrm{~nm})$} \\
\cline { 2 - 3 } Time $(\mathrm{h})$ & Periodate & Control \\
\hline 20 & 1.067 & 1.024 \\
5 & 1.012 & 1.088 \\
4 & 1.092 & 1.040 \\
3 & 1.131 & 0.988 \\
2 & 1.149 & 1.061 \\
1 & 1.108 & 0.971 \\
0.5 & 1.135 & 0.977 \\
\hline
\end{tabular}

${ }^{a}$ Each value represents the mean of replicated values. Least significant difference among means $(\alpha=0.001)=0.104$ nificant reduction in absorbance value, as determined by ELISA, when boiled, with a reduction in absorbance value experienced after only $5 \mathrm{~min}$ at $100^{\circ} \mathrm{C}$ (Fig. 2A).

Two protease treatments were employed, namely trypsin and pronase digestion of surface washings immobilized in microtiter wells. The protease trypsin reduced the binding of GP20 surface antigens to the DD8 MAb by over $50 \%$ after $5 \mathrm{~h}$ of exposure (Fig. 2B). Binding of the DD8 MAb to immobilized antigen was reduced by pronase treatment in a similar manner, except incubation of the antigen with pronase for more than $4 \mathrm{~h}$ had no additional effect (Fig 2B). There was no effect of periodate oxidation on binding of the DD8 MAb to immobilized surface antigens in ELISA (Table 3).
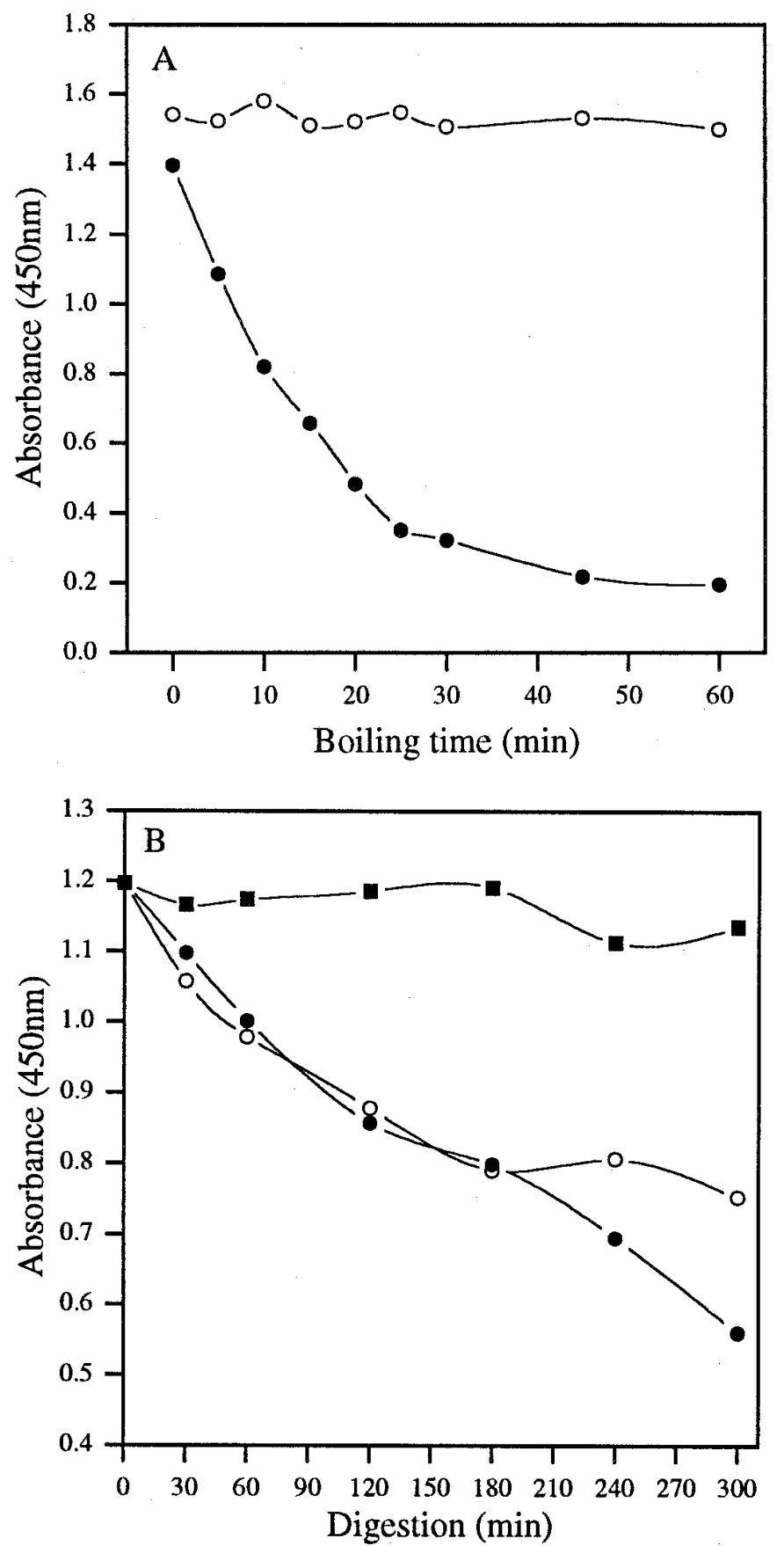

Fig. 2. A, Effect of boiling on binding of the DD8 monoclonal antibody (MAb) to immobilized Gaeumannomyces graminis var. tritici isolate GP20 antigens. -0 - = treated and -O- = control. B, Effect of protease treatment on binding of the DD8 MAb. $-\mathrm{O}-=$ pronase, $-\mathbf{-}-=$ trypsin and $-\mathbf{-}-=$ control. Each point (absorbance at $450 \mathrm{~nm}$ against incubation time in hours) represents the mean of duplicate samples. 
Promotion of antigen secretion by phenolic substrates. Secretion of the extracellular antigen recognized by the DD8 MAb was promoted by a number of phenolic compounds (Table 2; Fig. 3). Secretion of the antigen into liquid minimal medium was promoted by phenolic compounds meta-, ortho-, and para-cresol and 3,4,5-trihydroxybenzoic acid (gallic acid) and by L-(+)-ascorbic acid and L-DOPA. No increase in secretion was realized upon exposure to N,N'-bis (3,5-dimethoxy-4-hydroxybenzylidene) hydrazine (syringaldazine), a cresol derivative, or to 3-(4-hydroxyphenyl) alanine (L-tyrosine) compared to the negative control (minimal medium only). Tannic acid had an inhibitory effect on antigen secretion.

Inhibition of antigen secretion. Production of the extracellular antigen by G. graminis var. tritici in response to MM-LD was completely eliminated by the addition of EDTA, fusaric acid, 2-mercaptobenzothiazole (MERCAP), and 2-hydroxy-2,4,6-cycloheptatriene-1-one (tropolone) to the incubating medium (Fig. 4). Reductions in antigen production also were realized when kojic acid, pyroquilon, or diethyldithiocarbamic acid (DIECA) was added at a concentration of $100 \mathrm{mg} \mathrm{ml}^{-1}$ to the external medium compared to the positive control (minimal medium plus $500 \mathrm{mg}$ of L-DOPA per liter).

Secretion of antigen by other fungi. Promotion of antigen secretion by MM-LD, in fungi other than G. graminis var. tritici, was examined with single isolates of B. cinerea, $C$. globosum, $R$. solani, and $R$. cerealis (Fig. 5; Table 1). These represented the most cross-reactive of the species/isolates from secondary screening tests of antibody specificity (Fig 1B). Maximum antigen secretion, as determined by ELISA, was exhibited by all of the isolates after $4 \mathrm{~h}$ of incubation in MM-LD. Figure 6 represents the relative absorbance of each isolate as a percentage of the control (G. graminis var. tritici isolate GP20). There was no promotion of antigen secretion in the $R$. solani and $R$. cerealis isolates. Although there was no significant difference in absorbance values between $G$. graminis var. tritici and $B$. cinerea, promotion of antigen secretion in $G$. graminis var. tritici was significantly greater than that of C. globosum after $4 \mathrm{~h}$ of exposure to MM-LD.

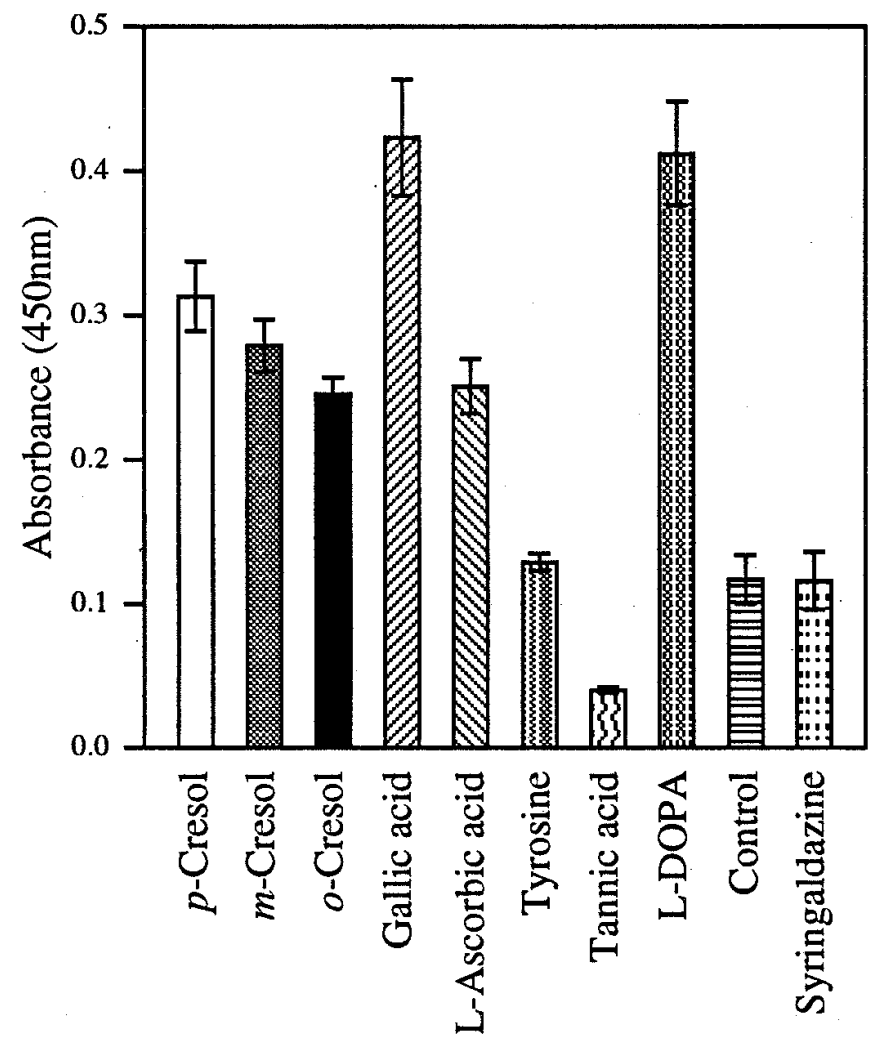

Fig. 3. Promotion of antigen secretion by phenolic substrates. Each bar (absorbance at $450 \mathrm{~nm}$ after $2 \mathrm{~h}$ of incubation) represents the mean of five replicate samples \pm standard error.
Recognition of purified laccase. The DD8 MAb exhibited strong recognition of the purified enzyme laccase in ELISA, with a limit of detection of $\sim 12 \mathrm{ng}$ of protein per ml of buffer (Fig. 6). There was no recognition of the control (purified tyrosinase).

IF. The MAb from cell line DD8 recognized both the broad melanized runner hyphae and more delicate hyaline mycelia of $G$. graminis var. tritici (GP20) by IF (Fig. 7A). No fluorescence was exhibited by the control (Fig. 7B).

\section{DISCUSSION}

Using surface antigens from live mycelia of the soilborne plant pathogen $G$. graminis var. tritici as the immunogen, we raised a hybridoma cell line secreting an IgM MAb, DD8, that recognizes surface antigens from G. graminis var. tritici, avenae, and graminis but that does not recognize antigens from a broad range of unrelated fungi, including Gliocladium roseum, Fusarium sp., Phoma exigua, Phialophora fastigiata, Penicillium crustosum, Pythium ultimum, Rhizopus stolonifer, Rhizoctonia carotae, $R$. oryzae, $R$. tuliparum, and $T$. viride. The MAb from this cell line, therefore, represents a considerable improvement in specificity compared to polyclonal antisera raised against $G$. graminis var. tritici in previous studies (15). Furthermore, the MAb raised in this study does not cross-react with surface antigens from a number of yeast species or gram positive and negative bacteria. The ability to discriminate between $G$. graminis var. tritici and other fungi, particularly species of Fusarium and Rhizopus, is a major advantage, because these fast-growing organisms have often proved to be the leading cause of failure to isolate $G$. graminis var. tritici from infected plant tissue $(1,21)$. The DD8 MAb cross-reacted with surface antigens from three Botrytis species, C. globosum, R. cerealis, and R. solani (Fig. 1B). Reasons for the cross-reactivity and procedures for the elimination of this nonspecific recognition are discussed below.

Characterization of the DD8 antigen by heat and protease treatments showed that the epitope recognized by the MAb was a protein. More specifically, sensitivity of the antigen to trypsin indicated that the MAb binds to a protein epitope that contains residues of

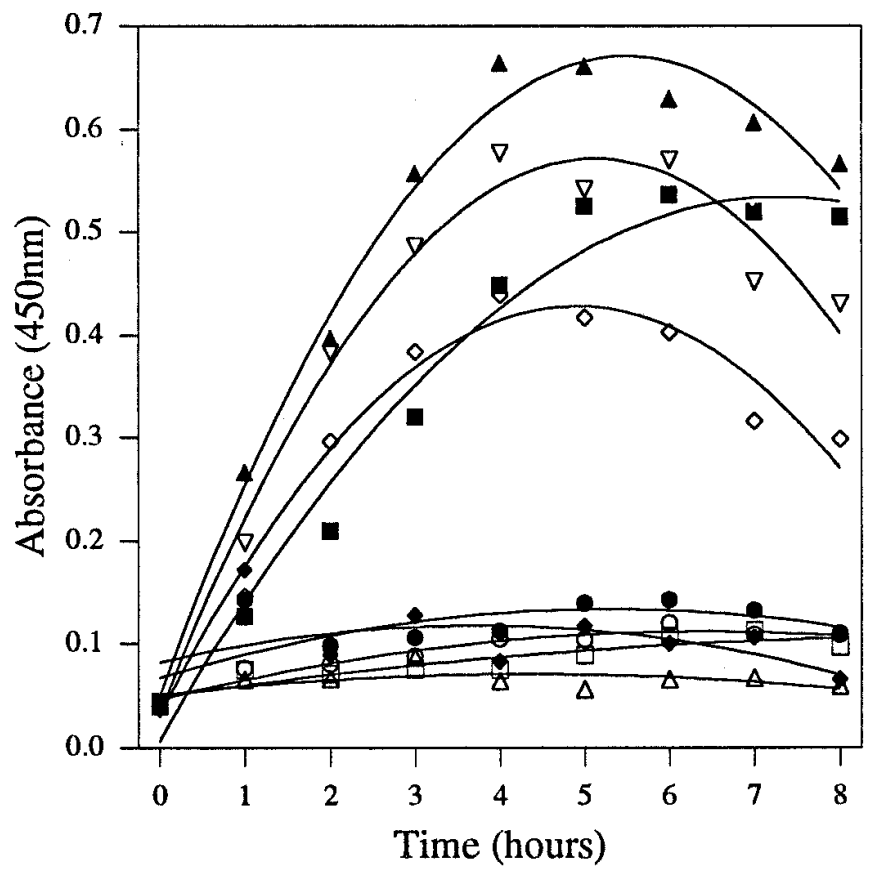

Fig. 4. Effect of inhibitors on promotion of antigen secretion by L-3-(3,4dihydroxyphenyl) alanine. $-\mathbf{\Delta}-=$ positive control; $-\nabla-=$ pyroquilon; $-\mathbf{-}-=$ DIECA; - $\diamond-=$ kojic acid; $-\diamond-=$ negative control; $-\mathbf{-}-=$ tropolone; $-\mathrm{O}-=$ fusaric acid; $-\triangle-=$ MERCAP; and $-\square-=$ EDTA. Each point represents the mean of four replicate samples. Curves were fitted with quadratic polynomials. 
the amino acids serine and arginine. The lack of effect of periodate oxidation on MAb binding to immobilized antigen implies that the epitope bound by the MAb does not contain a carbohydrate component. However, this result does not exclude the possibility that the protein epitope forms part of a larger glycoprotein molecule.

Antigen production, as determined by ELISA, was detected only in live mycelia, not in moribund mycelia. Moreover, secretion of antigen from quiescent tissue was promoted only by exposure of live mycelia to a select number of substrates, including monophenols $o^{-}, p-$, and $m$-cresol, benzoic acid gallic acid, phenolic amino acid L-DOPA, and antioxidant L-(+)-ascorbic acid. L-DOPA was used in the development of a semiselective medium for detection of G. graminis var. tritici (21) and is probably used by G. graminis var. tritici $(8,35)$ and other fungi for melanin biosynthesis. Indeed, exposure of $G$. graminis var. tritici to both L-DOPA and gallic acid led to rapid blackening of mycelia. Promotion of secretion of the DD8 antigen by these substrates suggests the extracellular, water-soluble antigen is either a product of a melanin biosynthetic pathway, an intermediate in a pathway, or, more importantly, a phenol oxidase enzyme such as tyrosinase or laccase.

Many fungi secrete extracellular enzymes that oxidize foreign phenolic metabolites and, in so doing, convert them into new polymers, most notably melanins $(16,25)$. Some fungi secrete peroxidase and hydrogen peroxide for this purpose (24), but this occurs only rarely in some species of the Basidiomycotina and not in the Ascomycotina to which G. graminis var. tritici belongs. Excluding a few cases of fungi in which tyrosinase is secreted extracellularly $(7,23)$, tyrosinase is an intracellular enzyme. In contrast, many reports on laccase describe its secretion by mycelia at one stage or another of the life cycle of fungi $(31,40)$. The DD8 MAb reacted strongly in ELISA with purified laccase from the rice blast fungus Magnaporthe grisea but did not recognize purified mushroom tyrosinase. Moreover, the antigen was not secreted into medium containing tyrosine. Laccases do not readily oxidize tyrosine. Interestingly, laccase secretion was not promoted by the cresol

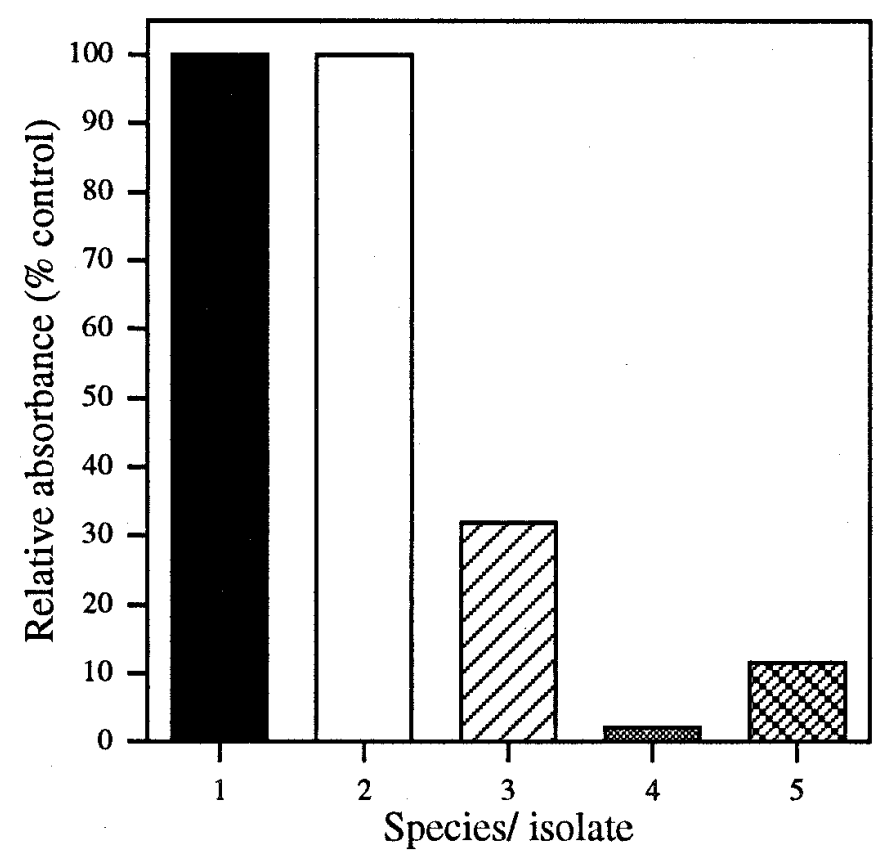

Fig. 5. Promotion of antigen secretion in Gaeumannomyces graminis var. tritici and other organisms by phenolic amino acid L-3-(3,4-dihydroxyphenyl) alanine (L-DOPA). 1, G. graminis var. tritici (isolate GP20); 2, Botrytis cinerea (isolate 2); 3, Chaetomium globosum (isolate 11); 4, Rhizoctonia solani (isolate 76124/22R01); and 5, R. cerealis (isolate SL4/1). Each bar (mean of triplicate values) represents the relative absorbance as a percentage of the control (G. graminis var. tritici [GP20]) after $4 \mathrm{~h}$ of incubation in minimal medium containing L-DOPA. derivative syringaldazine despite being considered uniquely a laccase substrate (17). However, this response also has been noted by other workers (20).

The evidence presented here strongly suggests the antigen recognized by the DD8 MAb is the enzyme laccase, and as such, the enzyme must represent an immunodominant molecule on the surface of G. graminis var. tritici hyphae. IF studies clearly showed that the enzyme is secreted by both the broad melanized runner hyphae (macrohyphae) and more delicate, hyaline mycelia of $G$. graminis var. tritici. Secretion of laccase by G. graminis var. trit$i c i$ also was demonstrated by the Bavendamm reaction (data not shown), whereby the development of a dark-brown zone around a colony of G. graminis var. tritici grown on PDA containing $10^{-3}$ $\mathrm{M}$ gallic acid or L-DOPA indicates the presence of an extracellular laccase (3). Differentiation of G. graminis var. tritici from other soilborne fungi on the basis of melanin pigment formation in the presence of L-DOPA was used by Juhnke et al. (21) in the development of a selective medium for the detection of the take-all pathogen.

All laccases investigated so far are glycoproteins $(28,29)$, and all contain copper. Consequently, reagents that interact with the copper in laccase can act as inhibitors of the enzyme (14). Such inhibitors include the specific copper chelators DIECA and MERCAP and other chelators less specific for copper, such as tropolone, EDTA, kojic acid, and fusaric acid. Fusaric acid, a toxin secreted by Gibberella fujikuroi, is a chelator of copper, cobalt, and iron and is an effective inhibitor of tyrosinase from various sources (6). Tropolone is one of the most potent inhibitors of mushroom tyrosinase (22). Tropolone, fusaric acid, EDTA, and MERCAP completely eliminated secretion of the DD8 antigen in response to MMLD, whereas DIECA and kojic acid also significantly reduced secretion from the fungus (Fig. 4). However, the reduction due to DIECA was only evident during initial periods of incubation in minimal medium containing L-DOPA. Interestingly, tannic acid also was inhibitory to antigen secretion by $G$. graminis var. tritici. The phenolic hydroxyl groups of tannins form stable cross-links with proteins, and as a result of this cross-linking, enzymes may be inhibited (38). These results strengthen the inference that the DD8 antigen is the polyphenol oxidase enzyme laccase. The antipene-

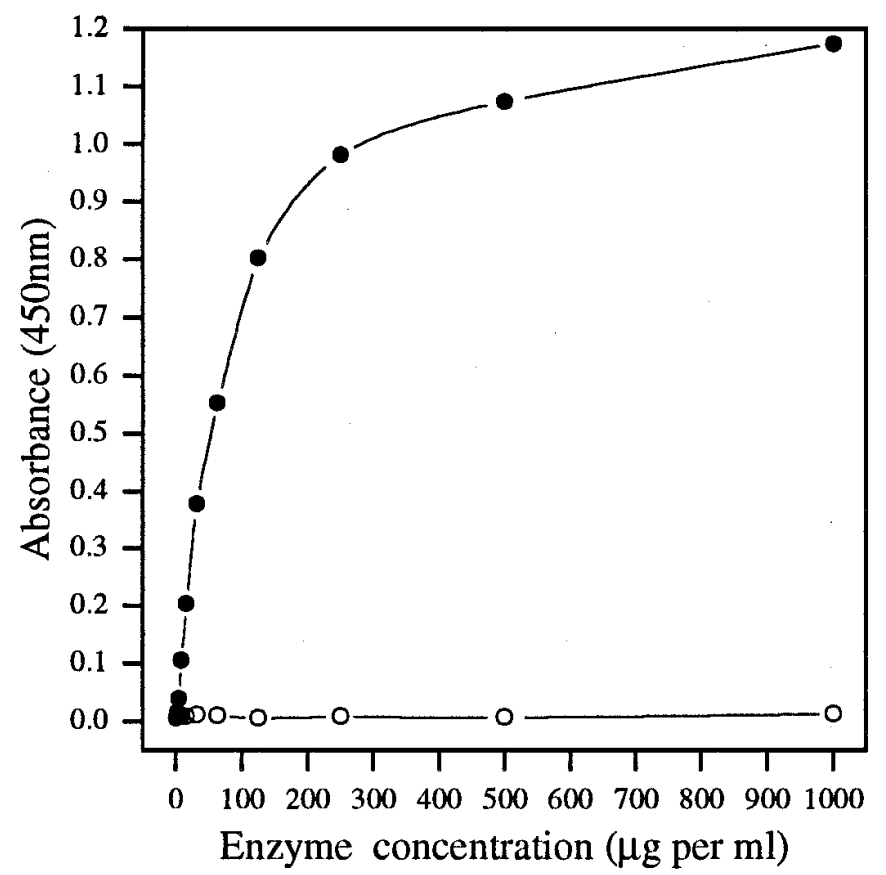

Fig. 6. Recognition of the purified enzyme laccase by the DD8 monoclonal antibody: - - laccase and -O- tyrosinase. Each point (absorbance at $450 \mathrm{~nm}$ against enzyme concentration in milligrams per liter) represents the mean of four replicate samples. 
trant fungicide pyroquilon is another compound that reduced secretion of the DD8 antigen. No firm inference is drawn as to the mode of action of pyroquilon on antigen secretion, but it inhibits 1,8-dihydroxynaphthalene (DHN)-melanin biosynthesis in Gaeumannomyces species (14) and other fungi (39).

A study of the literature reveals that laccase is present in a large number of fungi, including Ascomycetes, Basidiomycetes, and Fungi Imperfecti $(26,34)$. Secretion of laccases by wood-rot fungi and species of Botrytis and Rhizoctonia is well documented $(5,12,30)$, as is the ability of the soft-rot fungus $C$. globosum to degrade phenol derivatives (16). Several studies describe the isolation and characterization of cellulolytic enzymes produced by G. graminis var. tritici both in vitro and in vivo (11), but with the exception of Caesar-Tonthat et al. (8), no mention could be found of production of laccase by this fungus. Our work supports the premise that the laccase produced by G. graminis is the wall-bound enzyme involved in the synthesis of DHN-melanin (4) and the oxidation of L-DOPA in the outer wall of the fungus (4).

Initial screening tests for specificity of the DD8 MAb showed that it cross-reacted with surface antigens from $C$. globosum and with antigens from isolates of Botrytis spp., $R$. cerealis, and $R$. solani. This cross-reactivity was not surprising in light of the ubiquity of polyphenol oxidases in these fungi. Moreover, recent work in this laboratory (C. R. Thornton, unpublished data), using $R$. solani-specific MAbs raised in a separate study (32), has shown that in addition to laccase (36) $R$. solani excretes the enzyme catechol oxidase (EC 1.10.3.1) and that secretion is promoted by the benzoic acid gallic acid (32). The similarity between these enzymes could explain the cross-reactivity of the DD8 MAb with Rhizoctonia surface antigens. Further work will aim to clarify this relationship.

Use of the DD8 MAb to detect and differentiate between the target organism $G$. graminis, other common root and basal culm pathogens of small grains, such as $R$. cerealis and $R$. solani, and
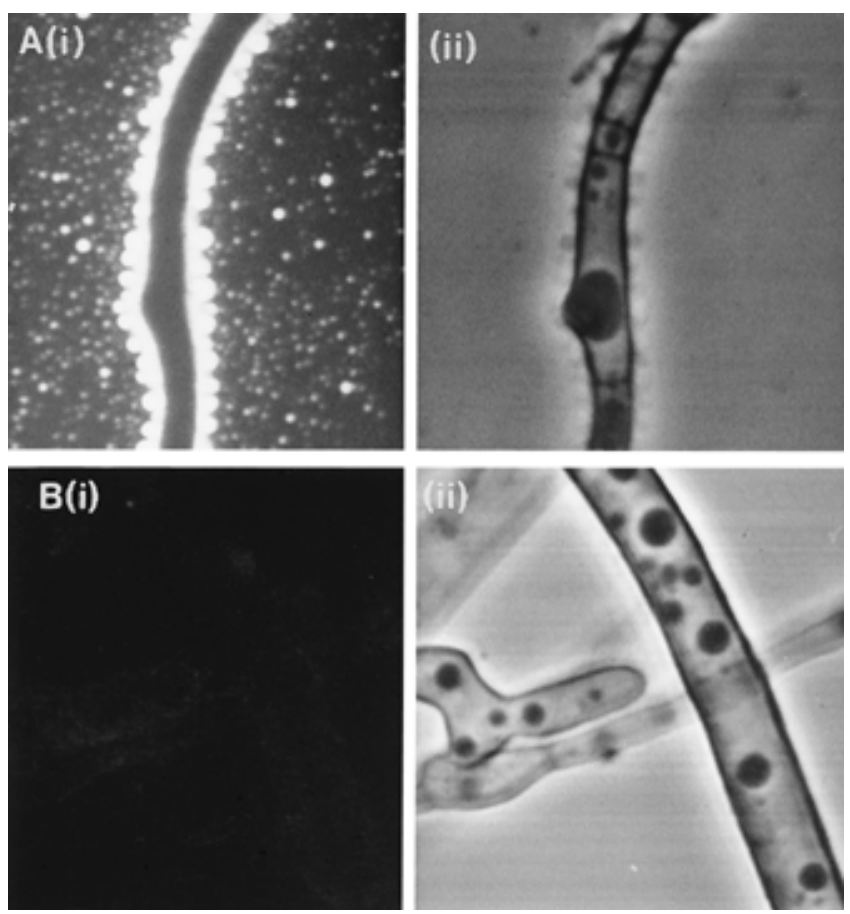

Fig. 7. A, Photomicrograph of Gaeumannomyces graminis var. tritici (isolate GP20) mycelium immunostained with monoclonal antibodies from the cloned cell line DD8 and anti-mouse immunoglobulin class M (IgM) ( $\mu$-chain specific) fluorescein isothiocyanate (FITC) conjugate examined under epifluorescence (i) and differential phase contrast (ii) transmission microscopy, respectively. B, Control. Mycelium incubated with tissue culture medium followed by anti-mouse IgM ( $\mu$-chain specific) FITC conjugate. $\times 400$ magnification; frame size $=30 \mu \mathrm{m}$. the soil-inhabiting fungi $C$. globosum and B. cinerea seemed, in the first instance, not feasible. However, further investigations clearly showed that although secretion of laccase by $B$. cinerea was promoted by L-DOPA, secretion in $R$. solani and $R$. cerealis was not promoted by this substrate, and secretion by $C$. globosum was significantly less compared to $G$. graminis var. tritici. Therefore, using a system of nutrient enrichment similar to that developed by Thornton et al. (32) for the detection of Rhizoctonia solani in soil, it may possible to differentiate live mycelia of $G$. graminis from cross-reactive Rhizoctonia spp. Cross-reactivity of the DD8 MAb with B. cinerea and $C$. globosum remains a problem, and methods to prevent or minimize nonspecific recognition are being investigated. For example, preliminary evidence suggests that $B$. cinerea polyphenol oxidase is unable to oxidize $p$-cresol. Future work will examine the range of phenolic compounds oxidized preferentially by $G$. graminis.

The DD8 MAb is currently being used to develop enzyme immunoassays for specific detection, quantification, and visualization of G. graminis var. tritici in soil and in planta. These will be used to monitor the spatio-temporal dynamics of G. graminis var. tritici in media of increasing microbiological complexity under defined laboratory conditions. The use of the DD8 MAb in combination with nucleic acid-based techniques, such as PCR, has enormous potential for rapid, sensitive and accurate detection of $G$. graminis var. tritici in naturally infested plant and soil samples.

\section{ACKNOWLEDGMENTS}

Imaging and analytical studies (confocal microscopy) were carried out at the Multi-Imaging Centre, School of Biological Sciences, University of Cambridge, which was established with support from the Wellcome Trust, U.K. Research supported by the Biotechnology and Biological Sciences Research Council (C. R. Thornton) and the Leverhulme Trust (F. M. Dewey).We thank P. Torok for his help with confocal microscopy and J. Tree for culture of hybridoma supernatants.

\section{LITERATURE CITED}

1. Asher, M. J. C. 1978. Isolation of Gaeumannomyces graminis var. tritici from roots. Trans. Br. Mycol. Soc. 71:322-325.

2. Asher, M. J. C. 1980 . Variation in pathogenicity and cultural characteristics in Gaeumannomyces graminis var. tritici. Trans. Br. Mycol. Soc. 75: 213-220.

3. Bavendamm, W. 1928. Ueber das Vorkomen und den Nachweis von Oxydasen bei holzzerstorenden Pilzen. Z. Pflanzenkrankr. Pflanzenshutz 38:257.

4. Bell, A. A., and Wheeler, M. H. 1986. Biosynthesis and functions of fungal melanins. Annu. Rev. Phytopathol. 24:411-451.

5. Bollag, J. M., Shuttleworth, K. L., and Anderson, D. H. 1988. Laccasemediated detoxification of phenolic compounds. Appl. Environ. Microbiol. 54:3086-3091.

6. Bossi, R. 1959. The influence of extracts of rotted apples on the browning of solutions of phenolic substances. Phytopathol. Z. 37:273.

7. Bruchet, G. 1966. Reserche de phenoloxydases myceliennes chez les Hebelomes. C. R. Acad. Sci. Paris 262:1940.

8. Caesar-Tonthat, T.-C., Van Ommen Kloeke, F., Geesey, G. G., and Henson, J. M. 1995. Melanin production by a filamentous soil fungus in response to copper and localization of copper sulfide by sulfide silver staining. Appl. Environ. Microbiol. 61:1968-1975.

9. Dewey, F. M. 1992. Detection of plant invading fungi by monoclonal antibodies. Pages 42-47 in: Techniques for the Rapid Detection of Plant Pathogens. J. M. Duncan and L. Torrance, eds. Blackwell Scientific Publications, Oxford.

10. Dewey, F. M., MacDonald, M. M., and Phillips, S. I. 1989. Development of monoclonal antibody-ELISA, -DOT-BLOT and -DIP-STICK immunoassays for Humicola lanuginosa in rice. J. Gen. Microbiol. 135:361-374.

11. Dori, S., Solel, Z., and Barash, I. 1995. Cell wall-degrading enzymes produced by Gaeumannomyces graminis var. tritici in vitro and in vivo. Physiol. Mol. Plant Pathol. 46:189-198.

12. Dubernet, M., Ribereau-Gayon, P., Lerner, H. R., Harel, E., and Mayer, A. M. 1977. Purification and properties of laccase from Botrytis cinerea. Phytochemistry 16:191-193.

13. Duffy, B. K., and Weller, D. M. 1994. A semiselective and diagnostic medium for Gaeumannomyces graminis var. tritici. Phytopathology 84: $1407-1415$. 
14. Elliot, M. L. 1995. Effect of melanin biosynthesis inhibiting compounds on Gaeumannomyces species. Mycologia 87:370-374.

15. El-Nashaar, H. M., Moore, L. W., and George, R. A. 1986. Enzymelinked immunosorbent assay quantification of initial infection of wheat by Gaeumannomyces graminis var. tritici as moderated by biocontrol agents. Phytopathology 76:1319-1322.

16. Haars, A., and Huttermann, A. 1980. Function of laccase in the white-rot fungus Fomes annosus. Arch. Microbiol. 125:233-237.

17. Harkin, J. M., Larsen, M. J., and Obst, J. R. 1974. Use of syringaldazine for detection of laccase in sporophores of wood rotting fungi. Mycologia 66:469-476.

18. Henson, J. M. 1992. DNA hybridisation and polymerase chain reaction (PCR) tests for identification of Gaeumannomyces, Phialophora and Magnaporthe isolates. Mycol. Res. 96:629-636.

19. Henson, J. M., Goins, T., Grey, W., Mathre, D. E., and Elliott, M. L. 1993. Use of polymerase chain reaction to detect Gaeumannomyces graminis DNA in roots and soil. Phytopathology 83:283-287.

20. Joel, D., Marbach, I., and Mayer, A. M. 1978. Laccase in Anacardiaceae. Phytochemistry 17:796.

21. Juhnke, M. E., Mathre, D. E., and Sands, D. C. 1984. A selective medium for Gaeumannomyces graminis var. tritici. Plant Dis. 68:233-236.

22. Kahn, V., and Andrawis, A. 1985. Inhibition of mushroom tyrosinase by tropolone. Phytochemistry 24:905-908.

23. Kocher, H. P., and Ettlinger, L. 1975. Extracellular tyrosinase in Streptomyces. Pathol. Microbiol. 42:248.

24. Koenigs, J. W. 1972. Production of extracellular hydrogen peroxide and peroxidase by wood-rotting fungi. Phytopathology 62:100-110.

25. Martin, J. P., and Haider, K. 1980. A comparison of the use of phenolase and peroxidase for the synthesis of model humic acid-type polymers. Soil Sci. Soc. Am. J. 44:983-988.

26. Mayer, A. M., and Harel, E. 1979. Polyphenol oxidases in plants. Biochem. 18:193-215.

27. Ophel Keller, K., Engel, B., and Heinrich, K. 1995. Specific detection of Gaeumannomyces graminis in soil using polymerase chain reaction. Mycol. Res. 99:1385-1390.

28. Perry, C. R., Smith, M., Britnell, C. H., Wood, D. A., and Thurston, C. F.
1993. The structure of laccase protein and its synthesis by the commercial mushroom Agaricus bisporus. J. Gen. Microbiol. 139:171-178.

29. Rehman, A. U., and Thurston, C. F. 1992. Purification of laccase I from Armillaria mellea. J. Gen. Microbiol. 138:1251-1257.

30. Shuttleworth, K. L., Postie, L., and Bollag, J. M. 1986. Production of induced laccase by the fungus Rhizoctonia praticola. Can. J. Microbiol. $32: 867-870$.

31. Smith, J. F., Claydon, N., Love, M. E., Allan, M., and Wood, D. A. 1989. Effect of substrate depth on extracellular endocellulase and laccase production of Agaricus bisporus. Mycol. Res. 93:292-296.

32. Thornton, C. R., Dewey, F. M., and Gilligan, C. A. 1993. Development of monoclonal antibody-based immunological assays for the detection of live propagules of Rhizoctonia solani in soil. Plant Pathol. 42:763-773.

33. Thornton, C. R., Dewey, F. M., and Gilligan, C. A. 1994. Development of a monoclonal antibody-based enzyme-linked immunosorbent assay for the detection of live propagules of Trichoderma harzianum in a peatbran medium. Soil Biol. Biochem. 26:909-920.

34. Thurston, C. F. 1994. The structure and function of fungal laccases. Microbiology 140:19-26.

35. Tschudi, S., and Kern, H. 1979. Specific lysis of the mycelium of Gaeumannomyces graminis by enzymes of Streptomyces lavendulae. Pages 611-615 in: Soil-borne Plant Pathogens. B. Schippers and W. Gams, eds. Academic Press, London.

36. Wahleithner, J. A., Xu, F., Brown, K. M., Brown, S. H., Golightly, E. J., Halkier, T., Kauppinen, S., Pederson, A., and Schneider, P. 1996. The identification and characterization of four laccases from the plant pathogenic fungus Rhizoctonia solani. Curr. Genet. 29:395-403.

37. Walker, J. 1975. Take-all diseases of Gramineae: A review of recent work. Rev. Plant Pathol. 54:113-144.

38. Walker, J. R. L. 1975. The Biology of Plant Phenolics. Edward Arnold Ltd., London.

39. Wheeler, M. H., and Greenblatt, G. A. 1988. The inhibition of melanin biosynthetic reactions in Pyricularia oryzae by compounds that prevent rice blast disease. Exp. Mycol. 12:151-160.

40. Wood, D. A. 1980. Inactivation of extracellular laccase during fruiting of Agaricus bisporus. J. Gen. Microbiol. 117:327-338. 